\section{Pontine tumour with central neurogenic hyperventilation}

Sir: Central neurogenic hyperventilation has for some time been suspected to arise from a dysfunction within the pontine reticular formation.' The evidence has, however, been subject to some controversy. ${ }^{2}$ I describe a patient with a clinical picture of a brain stem neoplasm, confirmed by MRI to be a pontine tumour, and with marked hyperventilation 'in the abscence of hypoxaemia or lung pathology.

The patient was a 48 year old man previously in good health. At the end of 1985 he noticed dizziness and some unsteadiness when making abrupt movements. He also complained of occipital headache of moderate intensity. After January 1987 several new symptoms emerged. The most notable one was a dyspnoea with forced breathing, described by his physician as a typical anxiety-provoked hyperventilation. He was referred to a psychologist, but after the next few months presented with diplopia and frequent micturition in addition to an exacerbation of his unsteadiness. When admitted to hospital in June 1987, he was found to have staccato eye movements with diplopia. Moderate dysarthria was present as well as marked atrophy and paresis of his right sternocleidomastoid muscle. There was pathological hyperreflexia in the lower extremities and the left-sided plantar reflex was extensor. His gait was moderately ataxic. The most impressive observation was, however, a marked overbreathing that was very unpleasant to the patient.

Routine blood samples were normal. However, analysis of his arterial blood gases revealed a profound hypocapnia of $1.7 \mathrm{kPa}$ (normal range 4.7-6.0) combined with an elevated arterial oxygen tension of $18 \cdot 1 \mathrm{kPa}$. Arterial $\mathrm{pH}$ was $7 \cdot 53$, Base Excess$12 \mathrm{mmol} / 1$, and actual $\mathrm{HCO}_{3} 11 \mathrm{mmol} / 1$, the latter values reflecting a partial metabolic compensation of his primary respiratory alkalosis. Chest radiographs were normal, as was the ECG and the EEG. Cerebral CT with special reference to the posterior fossa was undecisive. An MRI demonstrated, however, a definite expansion of the pons in both anteroposterior and right-to-left diameters, suggesting a glioma (fig).

Lumbar cerebrospinal fluid was normal including a protein content of $0.46 \mathrm{~g} / \mathrm{l}$ with a normal agarose gel electrophoresis. Visual evoked responses (VER) were normal. Brain stem auditory evoked responses (BAER) and somatosensory evoked responses (SER) were both abnormal with delays in central sonduction velocity.

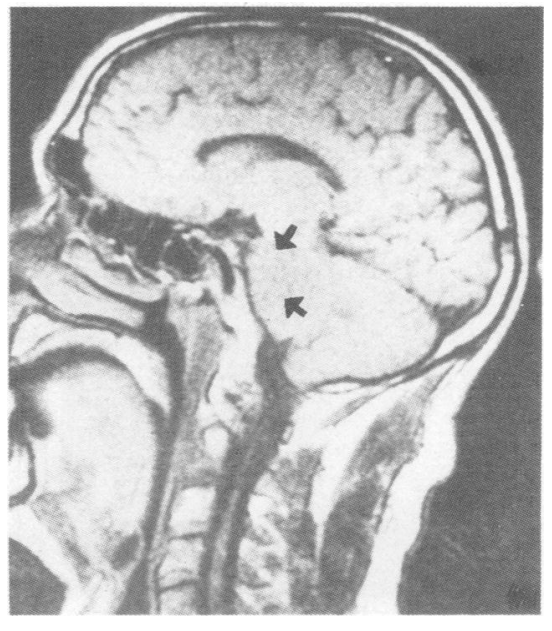

Fig MRI demonstrating a pontine expansion with varying densities within the tumour. Arrons indicate the lesion.

Spirometry disclosed a normal lung vital capacity of 4.90 litres with $F E V_{\text {, of }} 75 \%$ and peak expiratory flow (PEF) $460 \mathrm{l} / \mathrm{min}$. COsingle breath gas transfer factor was 8.9 , which is within the normal range, thus confirming a normal alveolo-capillary gas exchange. His breathing frequency was monitored continuously during wakefulness and sleep for 90 minutes, demonstrating a sustained tachypnoea of $22-24$ breaths/minute throughout the observation period, with no change during sleep.

Plum and Swanson inferred that lesions within the pontine reticular formation caused a neurogenic hyperventilation, presumably by interfering with inhibitory pathways to the medullary respiratory centres.' However, Plum and Posner were doubtful of this hypothesis in a later publication. ${ }^{2}$ They pointed out that all patients in the cited study were comatose, all had below normal oxygen tension levels, and at necropsy all cases had congested lungs. Thus, the hyperventilation might have been secondary to pulmonary congestion with hypoxia and reflex stimulation of the respiratory centres.

Lange and Laszlo reported a hyperventilating patient with a CNS tumour involving the pons and midbrain, but sparing the medulla oblongata. ${ }^{3}$ This 51 year old man had an extremely lowered arterial $\mathrm{CO}_{2}$ tension of $12 \mathrm{~mm} \mathrm{Hg}$ and an alkaline $\mathrm{pH}$ of 7.58. The oxygen saturation was cited as being $99 \%$. Interestingly, hyperventilation was the first sign of CNS dysfunction in this patient, noticed while he was still fully awake and conscious.

Goulon et al also described a patient who was fully awake with a central neurogenic hyperventilation; the diagnosis was a primary brain stem astrocytoma infiltrating both the medulla oblongata and the lower pontine tegmentum. ${ }^{4}$

In 1982 Rodriguez et al reported on a 53 year old woman with a brain stem astrocytoma demonstrated at necropsy to infiltrate the entire extent of the medulla oblongata as well as the tegmentum of the pons ${ }^{5}$ She remained alert and oriented for eight days while continuously hyperventilating with a respiratory frequency of about 22 breaths/minute, producing an arterial $\mathrm{CO}_{2}$ level of $9 \mathrm{~mm} \mathrm{Hg}$ and a $\mathrm{pH}$ of 7.74. Intravenously administered morphine reduced her breathing rate to $16-19 /$ minute and made the $\mathrm{CO}_{2}$ level increase to $21 \mathrm{~mm} \mathrm{Hg}$. She died from bilateral bronchopneumonia one week later.

A female patient with an extensive primary CNS lymphoma with no lesions below the level of the superior colliculi, and with a sustained tachypnoeic hyperventilation, was recently reported by Bateman et al. ${ }^{6}$ Her tachypnoea persisted during sleep, and blood gas analyses demonstrated a profound respiratory alkalosis of $7 \cdot 61$; the arterial $\mathrm{CO}_{2}$ tension was $1.6 \mathrm{kPa}$ and arterial $\mathrm{O}_{2}$ tension $15 \cdot 5 \mathrm{kPa}$.

Bateman suggests that a local acidosis due to an abnormal production of lactic acid in the brain stem neoplasm could explain the hyperventilation by a stimulation of chemoreceptors at the medullary level. However, recent in vivo studies measuring $\mathrm{pH}$ in brain neoplasms by the positron emission tomographic (PET) technique have demonstrated that $\mathrm{pH}$ in gliomas is higher than in the rest of the brain, thus ruling out the theory of local lactoacidosis as the sole cause of hyperventilation. ${ }^{7}$

Central neurogenic hyperventilation caused by a brain stem lesion is probably a very rare condition. To fulfil the criteria of this diagnosis, the arterial $\mathrm{CO}_{2}$ tension would have to be significantly decreased and arterial oxygen tension increased while breathing room air, the $\mathrm{pH}$ being at least moderately alkaline. This pattern should persist unaltered during wakefulness and sleep, and there should be no evidence of pulmonary congestion.

ROLF SALVESEN, Department of Neurology, Central Hospital of Nordland, 8017 Nordland Sentralsykehus, Norway 


\section{References}

1 Plum F, Swanson AG. Central neurogenic hyperventilation in man. Arch Neurol Psych (Chic). 1959;81:535-49.

2 Plum F, Posner JB. The pathologic physiology of signs and symptoms in coma. In: Plum F, Posner JB. eds. The diagnosis of Stupor and Coma. Philadelphia: FA Davis, 1980;36-8.

3 Lange LS, Laszlo G. Cerebral tumour presenting with hyperventilation. $J$ Neurol Neurosurg Psychiatry. 1965;28:317-9.

4 Goulon M, Escourolle R, Augustin P. Hyperventilation primitive par gliome bulboprotuberantiel. Rev Neurol (Paris) 1969; 121:636-9.

5 Rodriguez M, Baele PL, Marsh HM, Okazaki $H$. Central neurogenic hyperventilation in an awake patient with brain stem astrocytoma. Ann Neurol 1982;11:625-8.

6 Bateman DE, Gibson GJ, Hudgson P, Tomlinson $\mathrm{BE}$. Central neurogenic hyperventilation in a conscious patient with a primary cerebral lymphoma. Ann Neurol 1985;17:402-5.

7 Rottenberg DA, Ginos JZ, Kearfott KJ, Junck L, Dahwan V, Jarden JO. In vivo measurement of brain tumour $\mathrm{pH}$ using $11 \mathrm{c}-$ dimethyloxazolidine-dione and positron emission tomography. Ann Neurol 1984; 16:132-3.

Isolated schwannoma of the fourth cranial nerve: case report

Sir: Schwannomas of the nerves supplying the external ocular muscles are exceptionally rare. The literature contains only six reports of such a tumour arising from the trochlear nerve. A further case is reported here.

A 56 year old van driver was referred with a five month history of increasing weakness and clumsiness of his right limbs together with slurring of speech. For two weeks he had suffered from morning headaches and double vision on gaze to the rigit. Before the onset of his first symptoms he had been in good health. There was no family history of neurological disease.

Non neurological examination was unremarkable; he showed no features of neurofibromatosis. His mental state and optic discs were normal. He had a marked spastic dysarthria. There were no abnormalities of the cranial nerves other than a right lower facial weakness. In particular, he had full external ocular movements without diplopia and no trigeminal deficit. He had a moderately severe spastic right hemiparesis in the limbs. The tendon reflexes were symmetrically brisk and both plantar reflexes were extensor. He was only just able to walk without assistance.
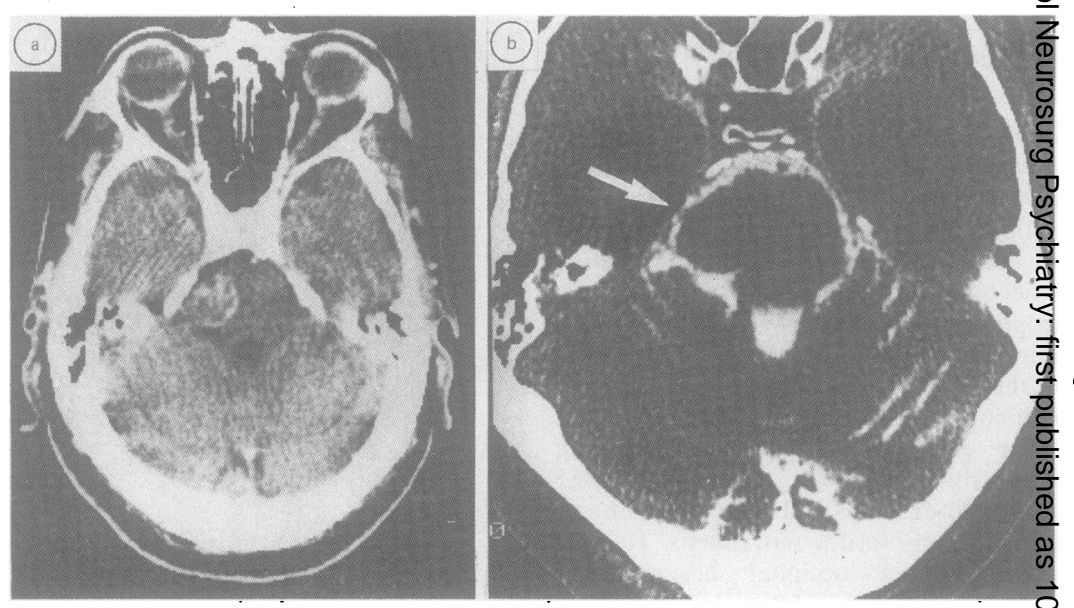

Fig a CT: after contrast enhancement. Shows oval well-defined lesion in the side of the brain-stem. b CT: with intrathecal contrast. Tumour (arrowed) is shown to be extrinsic to the brain-stem.

Skull radiographs showed no abnormality. Before enhancement the CT scan showed no abnormality. With contrast enhancement an oval irregularly hyperdense lesion $2 \mathrm{~cm}$ in diameter could be seen situated superficially in the left cerebral peduncle at the level of the tentorial edge (fig a). There was no distortion or displacement of the Aqueduct of Sylvius. CT after injection of intrathecal contrast showed that the mass was extrinsic rather than intrinsic (fig b).

The left cerebellar hemisphere was retracted via a left sided sub-occipital craniectomy, to permit inspection of the anterior part of the left cerebellopontine angle. A welldefined tumour $2 \mathrm{~cm}$ across was found partially embedded in the left side of the brain stem and lightly adherent to the back of the clivus at the level of the tentorium. The tumour lay above and medial to the left 7 th and 8th cranial nerves and the left trigeminal sensory root was stretched over its upper pole. The far side of the tumour abutted the right trigeminal sensory root. The tumour was separated from the adjacent structures and totally removed. Removal involved the sacrifice of a single nerve of small diameter which blended into the tumour capsule. Histological examination revealed a typical schwannoma.

Post-operative recovery was uneventful The patient complained of some inconstant double vision on gaze downwards and to the right with a tilted false image.

Two years later the patient had retired from work but was otherwise leading a full life with no disability. The patient showed the slight head tilt of a patient with a trochlear nerve palsy. His speech was nojy virtually normal and there was only a sligh degree of spasticity of the right limbs. He was able to walk briskly but with a degree of evident stiffness of the right leg.

Most intracranial schwannomas क्षrist from the 8th cranial nerve. Nexf frequency, after acoustic schwannomas,are the trigeminal variety but they only accur for $0.2 \%$ of intracranial tumours. ${ }^{1}$ Im $t$ the absence of von Recklinghausen's dis\&s, schwannomas of the other cranial nervat exceedingly rare. The first report of $\bar{P}$ isolated schwannoma of the 4th nerve was be King in $1976 .^{2}$ Including our patient, seveg cases have now been reported. ${ }^{2-6}$

All but two of the tumours arose from the 4th nerve where it passes round the side of the cerebral peduncle, close to the tentorif edge. In one patient the origin of the tumogr was probably more distal at or beyond the point where the nerve pierces the dura to enter the cavernous sinus, as the tumour la mainly on the medial aspect of the middie fossa floor. Only one tumour was close to the point where the nerve becomes invested wid schwann cells, a few millimetres after it has left the roof of the midbrain. Nevertheless, 3 t is widely believed that the schwannomas the cranial nerves tend to arise at the gliq. schwann cell junction, as they appear to do. the case of acoustic tumours. ${ }^{6}$

All but one of the patients were age between 32-58 years and five of the seven cases were women. The symptoms and signs resulted from a combination of dysfunction of the 4th nerve and compression of the adjacent cerebral peduncle and trigeminfy sensory root. The tumour with a middle 